\title{
MODELLING TWINNING IN TEXTURE DEVELOPMENT CODES
}

\author{
R. A. LEBENSOHN and C. N. TOME \\ IFIR - (CONICET - UNR) - 27 de Febrero 210 bis \\ 2000 Rosario - Argentina
}

\section{INTRODUCTION}

The plastic deformation of non-cubic materials is characterized by an intrinsic plastic anisotropy at the grain level and in most cases also by a substantial twinning activity. As a consequence, a reliable method for modelling twinning is required when simulating large strain deformation of these materials.

In this paper we are concerned with the treatment of the twinned volume fractions and with their contribution to texture. Since splitting the grain and treating the twinned fractions as new orientations leads soon to a numerically unmanageable number of grains, some approximation is needed. The approach proposed by Van Houtte ${ }^{[1]}$ is a Monte Carlo scheme based on replacing the original orientation by one of the twinning related orientations, according to a probabilistic criterion. In such a way, the total number of grains simulating the polycrystal is kept constant throughout deformation. In a recent paper ${ }^{[2]}$ we explore a Predominant Twinning Reorientation Scheme, based on a deterministic criterion: as the name suggests, the more active twinning system in the grain is chosen to reorient the whole grain. We conclude that neither the probabilistic nor the deterministic approach lead to realistic textures when applied to cases where twinning dominates deformation.

In what follows we will show that a Volume Fraction Transfer (VFT) Scheme is better suited to deal with such cases. The VFT method, originally proposed at ICOTOM-8 by Tomé et $a l^{[3]}$ and explored in detail in Ref. [2], is applied here to calculate texture development in zirconium deformed by rolling and Zircaloy bar deformed in tension and compression. In both cases, we are able to reproduce experimental deformation textures starting from an initially textured material. 


\section{THE VOLUME FRACTION TRANSFER SCHEME}

Within the VFT Scheme the polycrystal is represented by means of a discrete set of orientations with proper weights. However, the VFT Scheme differs from the classical models for texture development in that the orientations are kept fixed while the associated volume fractions are allowed to evolve during deformation. Within this scheme the Euler space is partitioned regularly in approximately equiaxed cells of $10^{\circ}$ by side (for the case of hexagonal symmetry this partition amounts to about 2000 cells). The orientations are made to coincide with the center of the cells and a certain volume fraction of material is assigned to each cell in correspondence with the initial texture. Plastic deformation is imposed to these representative grains assuming Fully Constrained conditions and the resulting reorientation can be visualized as a displacement in Euler space of the representative point. We assume that the cell as a whole displaces rigidly by that amount and that the material is uniformly distributed within it. When displaced, the cell partially overlaps with the neighboring cells and the volume fraction of material contained in the overlapped portions is substracted from it and 'transferred' to the neighboring cells. This process of transference, repeated after every strain increment, leads to a gradual variation of the volume fraction in each cell and so to texture development. When twinning is active, a fraction of the grain adopts a new orientation and is transferred to the corresponding cell in Euler space and accumulated to the fraction already in the cell. By doing this we manage to account exactly for every twinned fraction in every twin system of every grain after each strain increment. For those materials which deform only by slip, we verify that the VFT Scheme predicts the same texture as the classical schemes, while for those cases where twinning prevales very different textures are obtained ${ }^{[2]}$.

\section{RESULTS AND DISCUSSION}

We calculate texture evolution in two materials which twin profusely and for which there is detailed information of texture development as a function of deformation.

The first case is a rolling experiment performed by Tenckhof $\mathrm{f}^{[4]}$ on a Zirconium sample which had been previously heat treated in order to achieve a coarse grain structure. The polycrystal was formed by about 20 large grains which were labeled, and deformation was imposed in several steps. After each step, not only the distribution of basal poles, but also the active slip and twinning systems were characterized in each grain. 
Tenckhoff reports profuse twinning activity, with secondary and higher order twins being observed in most of the grains. He identifies $\{10 \overline{1} 2\}<\overline{1} 011>$ (and less frequently $\{11 \overline{2} 1\}<\overline{1} \overline{1} 26>$ ) tensile twins, $\{11 \overline{2} 2\}<\overline{1} \overline{1} 23>$ compressive twins and $\{10 \overline{1} 0\}<11 \overline{2} 0>$ prism slip. The initial orientations and basal pole figures measured after $2 \%$, $18 \%, 45 \%$ and $73 \%$ true strain are shown in Fig. 1a. At $18 \%$ deformation a texture component starts forming along the ND; further deformation up to $45 \%$ reinforces such component and at 73\% the basal poles show the characteristic split towards the TD.

For the calculation with the VFT Scheme we reproduce the initial texture using the 18 labeled orientations shown in Fig.1a. Weights are assigned using Fig.1 in Tenckhoff's paper to infer the relative volume of the labeled grains. Since no information is provided concerning the prism poles, their starting orientations are randomly assigned. The simulation is performed using the active systems reported by Tenckhoff: $\{10 \overline{1} 0\}<11 \overline{2} 0>$ prism slip, $\{10 \overline{1} 2\}<\overline{1} 011>$ tensile twins and $\{11 \overline{2} 2\}<\overline{1} \overline{1} 23>$ compressive twins. A ratio of CRSS's of $1: 2: 2$ is assumed respectively, but the results are not changed if higher values of CRSS are assigned to the twinning systems ${ }^{[2]}$. The results of the simulation are presented in Fig. 1b. At $2 \%$ deformation the basal pole figure is not changed much with respect to the initial one but after $18 \%$ strain the simulated texture has developed faster than the experimental one and the ND component is already clearly formed. Further deformation up to $45 \%$ starts showing the splitting of the component towards the TD, a feature which appears in the experimental pole figure after $73 \%$ strain. As a conclusion: the VFT Scheme is able, starting with only 18 orientations, to keep track of the twinned fractions as deformation proceeds and to reproduce the observed development of texture for a case where twinning is the only reorientation mechanism. The classical schemes, based on reorienting the whole grain, do not predict a stable texture under such stringent conditions.

For the second application of the VFT Scheme we use results reported by MacEwen et al. ${ }^{(5)}$, of tensile and compressive experiments performed on cylindrical samples cut along the axis of a Zircaloy-2 bar. The samples were deformed up to $15 \%$ true strain in tension and up to $20 \%$ in compression, with an interruption after $8 \%$ strain in the latter case. The initial, intermediate and final distributions of basal (0002) and prismatic $\{1120\}$ and $\{10 \overline{10}\}$ poles are reproduced in Fig.2. Because of the cylindrical symmetry, pole intensities are reported as a function of the tilting angle $\theta$ instead of using level lines. The initial texture is typical of a swagged bar, with the basal poles mainly perpendicular to the bar axis and randomly distributed in the 
section of the bar. As was to be expected, since tensile deformation is close to the fabrication strain, it leaves the initial texture invariant after $15 \%$ deformation (see Fig. 2). Compression, however, induces tensile twinning activity, which tends to align the basal poles along the rod axis. The distribution of prismatic poles is also modified (see Fig. 2).

For the purpose of the simulation, weights are assigned to the cells in Euler space which reproduce the experimental pole distributions (see Fig. 2). Prismatic slip and $\{10 \overline{1} 2\}<\overline{1} 011>$ tensile twins are known to be active in Zircaloy-2 but, since compressive twins are not observed in this material, $\{10 \overline{1} 1\}<11 \overline{2} 3\rangle$ pyramidal slip is assumed to be present. A CRSS ratio of $1: 2: 2$ is assigned to the respective systems, although the same results are obtained for a wide range of CRSS's ${ }^{[2]}$. A quantitative comparison between the measured and the predicted pole intensities displayed in Fig.2 indicates that, except for a weaker basal pole component along the rod axis predicted after $20 \%$ compressive strain, the basal and prism pole distributions at $8 \%$ compressive and $15 \%$ tensile strain are in quite good agreement with the experimental counterpart.

\section{CONCLUSIONS}

We show in this work the advantage of using a Volume Fraction Transfer Scheme for simulating texture development in materials where twinning is the dominant deformation mechanism. By using fixed orientations in Euler space, this method permits not only to keep track exactly of the twinned volume fractions in every grain, but also to simulate reorientation due to slip. The agreement between our predictions and detailed experimental results indicates that the method is reliable and sound.

\section{REFERENCES}

[1] P. Van Houtte, Acta metall. 26 (1978) 591.

[2] C. N. Tomé, R. A. Lebensohn and U. F. Kocks, submitted to Acta metall.

[3] C. N. Tomé, G. R. Canova, S. Ahzi and A. Molinari, Proceedings of ICOTOM-8, J. Kallend and G. Gottsteln eds., The Metallurglcal Soc. (1988) 395.

[4] E. Tenckhoff, Metall. Trans. 9A (1978) 1401.

[5] S. R. MacEwen, N. Christodoulou, C. Tomé, J. Jackman, T. M. Holden, J. Faber Jr. and R. L. Hitterman, same as Ref. [3], 825. 


\section{ROLLING}

A) Experimental $[0002]$ poles
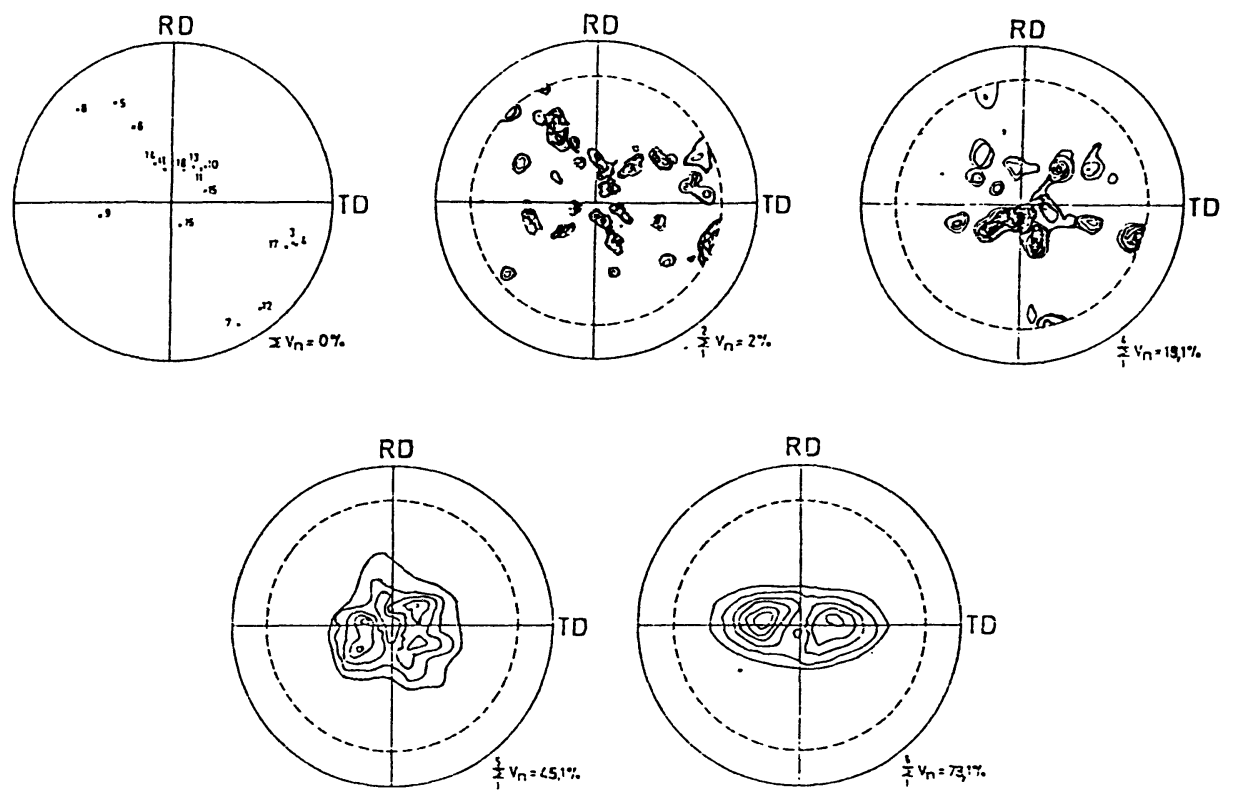

B) Calculated $[0002]$ poles
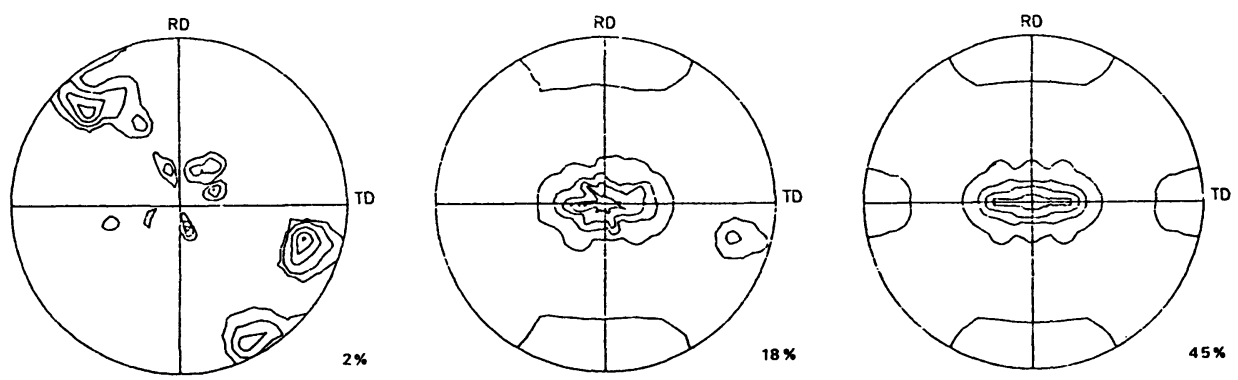

Figure 1: a) Initial basal pole orientations and basal pole figures measured by Tenckhof $\mathrm{f}^{[4]}$ in $\mathrm{Zr}$ after several deformation steps in plane strain compression. b) Basal pole distributions predicted with the VFT Scheme. 

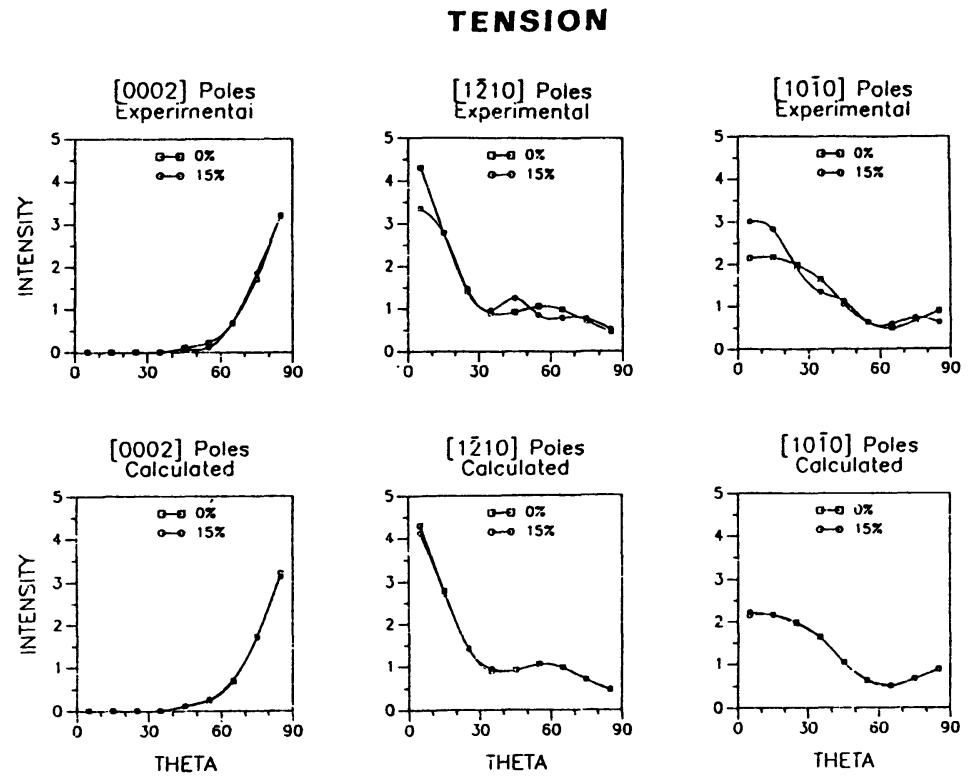

\section{COMPRESSION}
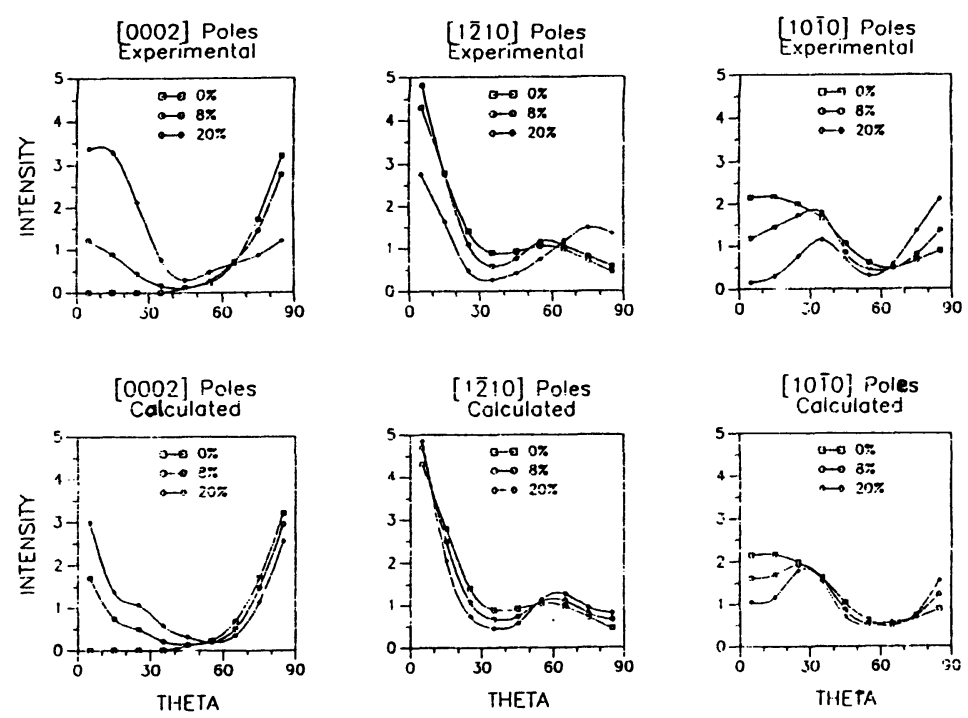

Figure 2: a) Meassured distribution of basal and prism poles after tensile and compressive deformation of Zircaloy-2 bar $^{[5]}$ and distributions predicted with the VFT Scheme for the same deformations. 